of the ocean to the ecosystems. Afterwards, however, Longhurst divides his book into as many chapters as there are provinces of the oceans (about 50!). The result is a long, tedious enumeration of descriptions of regions, each time with the same plan and, inevitably, often the same content. After all, every province is almost as complex as the entire ocean, and this complexity can hardly be described in three or four pages. For example, Longhurst makes the Gulf of California a subdivision of the California Current Province, but he recognizes that it could as well be divided into 14, or even 33 locations, taking it beyond the reach of this book's format.

In 1,000 years, one drop of ocean water may pass through most of the provinces. The author thinks that "the debate between partitions and a continuum is somewhat sterile because both concepts will be required for different purposes, and they are not mutually exclusive". To me, the continuum sounds much more exciting.

In reading this book, I have realized how much the generation time of grazers varies with latitude, and the consequences this has for the food chain. This alone would have made a nice chapter, but I had to pick such pieces of information from here and there throughout the 50 provinces.

The division into provinces also results in some oddities. Should we consider that the region of the Pacific Ocean that is affected by the El Niño has moving boundaries? The response time of most zooplankters in this region is much less than the duration of $\mathrm{El}$ Niño events, and the distribution of tuna changes during these events, so the answer should be yes. Why not consider, then, that the boundary between the North Atlantic Subtropical Gyral Province and the North Atlantic Drift Province moves similarly, with the northward shift of the summer thermocline? Indeed, many grazers in these provinces have short generation times. The boundaries proposed here are subject to endless discussion.

If this book is tedious to read, it is extremely well documented and precisely written, and this is remarkable considering the diversity of sources and sometimes the contradictions Longhurst had to work with.

In the tropical Atlantic and Pacific, my own area of expertise, I was surprised that there was no mention of the tropical instability waves in the Atlantic, and that the Guinea Dome was placed in the Western Tropical Atlantic Province while its analogue in the south, the Angola Dome, was placed in the Eastern Tropical Atlantic Province. Overall, however, the summaries that are given for these two large regions are precise and exhaustive.

Everyone will find something of interest in this book. Finally, we oceanographers like to give a name to the areas where we go on cruises. From this point of view, the partition proposed here is certainly the best one we have today.

Yves Dandonneau is at the Laboratoire d'Oceanographie Dynamique et de Climatologie, 4 place Jussieu, 75252 Paris cedex 05, France.

\section{Loose round-up lacks even circular logic}

\section{A History of the Circle \\ by Ernest Zebrowski Jr \\ Rutgers University Press: 1999. 214 pp. \$28 \\ lvor Grattan-Guinness}

Popular books on or around mathematics appear quite regularly nowadays. This one nominally takes the circle as its theme, both for its mathematical properties and for its use in the physical sciences. Based upon teaching in American colleges, the level is fairly elementary.

The early chapters treat several manifestations of the circle in antiquity, including the use of rollers in transporting heavy objects and some features of astronomy. But after that the circle gives way to conic sections and closed but non-circular orbits as studied from Kepler to Newton. It comes back later rather indirectly via oscillations, pendular and simple harmonic motions and waves, and associated mathematical theories such as Fourier series. Late chapters skim across relativity theory and quantum mechanics, sandwiching an interlude on ancient architecture.

While the emphasis on applications is well taken, the overall impression is rather incoherent: links to circularity are tenuous in places, and many topics specifically tied to circles are omitted. The most obvious case concerns $\pi$. Some properties are stated, but no highlight is given to its four main roles, of relating the diameter of a circle not only to its

\section{New in paperback}

\section{Countdown: A History of Space Flight}

by T. A. Heppenheimer

Wiley, £13.99, \$17.95

“... The history of spaceflight is unfinished. So too is Heppenheimer's book; the publisher left the last page blank, inadvertently one assumes. Authors sue for such incompetence, but in this case it might be a fitting conclusion to this useful but necessarily inconclusive study". Alex Roland, Nature 392, 143-145 (1998)

\section{The Covenant of the Wild: Why} Animals Chose Domestication by Stephen Budiansky

Yale University Press, $\$ 14.95$

"Budiansky's thesis is that human relationships with other species are not so much the 'exploitation' they are often made out to be, but circumference (hence the eighteenth-century choice of the letter $\pi$, for perimetros) but also to its area, and to the surface area and volume of the sphere. It is enlightening, and also not at all obvious, that the same factor $\pi$ is involved. The enabling theorem for the circle - area $=($ circumference $/ 2) \times($ diameter $/ 2)$ - is a profound result; the author presents one intuitive argument for it but does not register its significance. All sorts of other cultural possibilities are ignored; for example, that the classical problem in Greek geometry of squaring the circle may echo a desire, very evident with the Egyptians, of uniting the circular heavens with the Earth, long symbolized by the square.

As for history, the author states at once that he gives no conventional version. The accuracy of his statement can be fairly judged by a few typical quotations. "It was a long time before anyone had a practical need to measure angles to a precision much finer than one degree." In his Elements, Euclid "laboriously proved every mathematical relationship that was then known". "What Descartes did accomplish was to use rectangular co-ordinates in a new way" (he actually introduced analytic geometry). Einstein's creation of special relativity in 1905 was effected by "conduct[ing] his Gedanken experiments through mathematical logic"; indeed, in general "Scientists depend upon mathematical logic to generate their theoretical predictions" ("If only they did," Bertrand Russell might have sighed). Another circular sign comes to mind: 0 per cent for accuracy.

A good heuristic and historical introduction to parts of mathematics using the circle as its theme would make an excellent contribution to the popular understanding of mathematics. Perhaps somebody can take up the challenge.

Ivor Grattan-Guinness is at the Middlesex University, Queensway, Enfield, Middlesex EN3 4SF, UK. arise from quite natural coevolutionary processes like those between other pairs of species .. Perhaps the strength of the book is that it raises issues and makes points and then doesn't tell one what conclusions to draw." Marian Stamp Dawkins, Nature 356, 487 (1992)

Science, Jews \& Secular Culture: Studies in Mid-Twentieth-Century American Intellectual History by David A. Hollinger

Princeton University Press, $\$ 14.95, £ 12.50$

Re-enchanted Science: Holism in German Culture from Wilhelm II to Hitler

by Anne Harrington

Princeton University Press, \$19.95, $£ 11.95$ 